Type: Research Paper

Section: Color

\title{
Structural colour in Tersina viridis
}

\section{Color estructural en Tersina viridis}

\author{
C. D'Ambrosio ${ }^{1 *}$, M. Inchaussandague ${ }^{1,2}$, D. Skigin ${ }^{1}$, A. Barreira ${ }^{3}$, P. Tubaro ${ }^{3}$ \\ 1. Grupo de Electromagnetismo Aplicado, Departamento de Física,
}

Facultad de Ciencias Exactas y Naturales, Universidad de Buenos Aires, (C1428EGA), Buenos Aires, Argentina;

2. CONICET-UBA, Instituto de Física de Buenos Aires (IFIBA), Buenos Aires, Argentina

3. División de Ornitología, Museo Argentino de Ciencias Naturales, "Bernardino Rivadavia",

Av. Ángel Gallardo 470 (C1405DJR), Buenos Aires, Argentina.

${ }^{(*)}$ E-mail: chdambrosio@gmail.com

Received: 02/12/2016 Accepted: 01/08/2017

DOI: 10.7149/OPA.50.3.49058

\begin{abstract}
:
The colour of birds' plumage is due to the presence of pigments as well as to frequency selective structural effects produced by the interaction of light with the photonic microstructure that constitutes the feather barbs. The species Swallow Tanager, of the family Thraupidae, exhibits sexual dichromatism: males have blue plumage and females green. In addition, the feathers show an iridescent reflectance, which changes as the angle between the directions of incidence and observation is varied. In order to investigate the mechanisms of colour generation in the feathers of the Swallow Tanager species, we study their electromagnetic response. First, we obtained TEM images of the barbs' cross section, which revealed a microstructure consisting of quasi-spherical air cavities embedded in a $\beta$-keratin matrix. To obtain the relevant geometric parameters of the structure, we performed a detailed statistical analysis of the images. These parameters, together with the optical properties of the constituent materials, determine the electromagnetic behaviour of the structure. To calculate the reflectance of a barb, we used two theoretical approaches. First, we applied a simplified model based on Fourier analysis, which relies on the single-particle scattering hypothesis. This method provides useful information about the spectral position of the dominant reflectance peak in the visible region of the spectrum. However, this approach does not account for multiple scattering effects which could produce additional features. Then, we investigate the optical response by applying the Korringa-Kohn-Rostoker (KKR) method, which is a rigorous theoretical formalism for the calculation of the reflectance of structures formed by a stack of spheres embedded in a material medium. We performed numerical simulations, paying special attention to the influence of the geometrical characteristics of the microstructure on the optical response of the barbs. The KKR curves exhibit an enhancement in the UV region, which is in agreement with the measured reflectance of Swallow Tanager. These results evidence the potential of the KKR formalism to accurately reproduce the electromagnetic response of natural structures and to reveal features that cannot be explained using the Fourier approach. The results obtained confirm that the quasi-ordered spongy structure produces coherent scattering and therefore is the responsible for the colour of the Swallow Tanager.
\end{abstract}

Key words: structural colour, 3D photonic crystals, iridescence

\section{RESUMEN:}

El color del plumaje de las aves se debe tanto a la presencia de pigmentos como a efectos estructurales selectivos en frecuencia producidos por la interacción de la luz con la microestructura fotónica que constituye las barbas de las plumas. El plumaje de la especie Tersina viridis, de la familia Thraupidae, presenta dicromatismo sexual: los machos tienen plumaje azul y las hembras son verdes. Además, las plumas muestran una reflectancia iridiscente que varía con el ángulo entre la dirección de incidencia y la de observación. Para investigar los mecanismos de generación de color en las plumas de Tersina viridis estudiamos su respuesta electromagnética. En primer lugar, se obtuvieron 
imágenes TEM de la sección transversal de las barbas, las cuales revelaron una microestructura formada por cavidades cuasi-esféricas de aire inmersas en una matriz de $\beta$-queratina. Para obtener sus parámetros geométricos relevantes, se realizó un análisis estadístico detallado de las imágenes. Dichos parámetros, junto con las propiedades ópticas de los materiales involucrados, determinan el comportamiento electromagnético de la estructura. Para calcular la reflectancia de la barba se utilizaron dos métodos teóricos. En primer lugar, se aplicó un modelo simplificado basado en el análisis de Fourier que sólo tiene en cuenta eventos de esparcimiento simple. Este método provee información útil acerca de la posición espectral del pico de reflectancia dominante en la zona visible del espectro. Sin embargo, este análisis no considera las interacciones entre las esferas, las cuales podrían afectar significativamente la respuesta óptica. Para tener esto en cuenta, se aplicó el método conocido como Korringa-Kohn-Rostoker (KKR), que es un formalismo teórico riguroso para el cálculo de la reflectancia de estructuras formadas por apilamientos de esferas inmersas en un medio material. Se realizaron simulaciones numéricas prestando especial atención a la influencia de las características geométricas de la microestructura. Las curvas obtenidas muestran un pico de reflectancia en la zona del UV, tal como muestran las mediciones de los espectros de reflectancia de la especie. Estos resultados muestran el potencial del método KKR para reproducir en forma precisa la respuesta electromagnética de estructuras naturales y para reproducir características que no pueden ser explicadas utilizando el método de Fourier. Los resultados obtenidos confirman que la estructura fotónica cuasi-periódica de la barba produce esparcimiento coherente y por lo tanto es la responsable del color de la especie Tersina viridis.

Palabras clave: color estructural, cristales fotónicos 3D, iridiscencia

\section{REFERENCES AND LINKS / REFERENCIAS Y ENLACES}

[1] S. Kinoshita, Bionanophotonics: an introductory textbook, Pan Stanford Publishing Pte. Ltd. (2013). https://doi.org/10.1201/b15260

[2] S. Berthier, Iridescences, the physical colours of insects, Springer Science+Business Media, LLC (2007).

[3] V. Saranathan, J. Forster, H. Noh, S. Liew, S. Mochrie, H. Cao, E. Dufresne, R. Prum, "Structure and optical function of amorphous photonic nanostructures from avian feather barbs: a comparative small angle X-ray scattering (SAXS) analysis of 230 bird species," J. R. Soc. Interface 9, 2563-2580 (2012). https://doi.org/10.1098/rsif.2012.0191

[4] R. Prum, R. Torres, S. Williamson, J. Dyck, "Coherent light scattering by blue feather barbs," Nature 396, 28-29 (1998). https://doi.org/10.1038/23838

[5] R. Prum, R. Torres, S. Williamson, J. Dyck, "Two-dimensional Fourier analysis of the spongy medullary keratin of structurally coloured feather barbs," Proc. R. Soc. Lond. B 266, 13-22 (1999). https://doi.org/10.1098/rspb.1999.0598

[6] H. Noh, S. Liew, V. Saranathan, R. Prum, S. Mochrie, E. Dufresne, H. Cao, "Contribution of double scattering to structural coloration in quasiordered nanostructures of bird feathers," Phys. Rev. E 81, 051923 (2010). https://doi.org/10.1103/PhysRevE.81.051923

[7] A. Barreira, G. García, D. Lijtmaer, S. Lougheed, P. Tubaro, "Blue males and green females: sexual dichromatism in the Blue Dacnis (Dacnis cayana) and the Swallow Tanager (Tersina viridis)," Ornitología Neotropical 19, 441-450 (2008).

[8] A. Barreira, N. García, S. Lougheed, P. Tubaro, "Viewing geometry affects sexual dichromatism and conspicuousness of noniridescent plumage of Swallow Tanager (Tersina viridis)," The Auk 133, 530543 (2016). https://doi.org/10.1642/AUK-15-170.1

[9] A. Modinos, "Scattering of electromagnetic waves by a plane of spheres-formalism," Physica A 141, 575-588 (1987). https://doi.org/10.1016/0378-4371(87)90184-1

[10] N. Stefanou, V. Yannopapas, A. Modinos, "Heterostructures of photonic crystals: frequency bands and transmission coefficients," Comput. Phys. Commun. 113, 49-77 (1998). https://doi.org/10.1016/S0010-4655(98)00060-5 
[11] V. Yannopapas, A. Modinos, N. Stefanou, "Optical properties of metallodielectric photonic crystals," Phys. Rev. B 60, 5359 (1999). https://doi.org/10.1103/PhysRevB.60.5359

[12] N. Stefanou, V. Yannopapas, A. Modinos, "Multem 2: a new version of the program for transmission and bandstructure calculations of photonic crystals," Comput. Phys. Commun. 132, 189-196 (2000). https://doi.org/10.1016/S0010-4655(00)00131-4

[13] A. Barreira, Evolución de los patrones de coloración del plumaje en fruteros neotropicales, PhD Thesis, UBA (2011).

[14] ImageJ, https://imagej.nih.gov/ij/

[15] R. Prum, R. Torres, "A Fourier tool for the analysis of coherent light scattering by bio-optical nanostructures," Integr. Comp. Biol. 43, 591-602 (2003). https://doi.org/10.1093/icb/43.4.591

[16] F. Güller, Estudio de la respuesta electromagnética de cristales fotónicos formados por arreglos periódicos de esferas, Tesis de Licenciatura, UBA (2011).

[17] N. Ashcroft, N. Mermin, Solid State Physics, Philadelphia, Cengage Learning (1976).

[18] L. Dorado, R. Depine, G. Lozano, H. Míguez, "Interplay between crystal-size and disorder effects in the high-energy optical response of photonic crystal slabs," Phys. Rev. B 76, 245103 (2007). https://doi.org/10.1103/PhysRevB.76.245103

[19] L. Dorado, R. Depine, "Modelling of disorder effects and optical extinction in three-dimensional photonic crystals," Phys. Rev. B 79, 045124 (2007).

https://doi.org/10.1103/PhysRevB.79.045124

\section{Introduction}

Coloration in animals is produced by pigments, by nanostructures, or by a combination of both. Pigments produce colour by selectively absorbing specific wavelengths of visible light while allowing others to be reflected. On the other hand, structural colours are produced by periodic nanostructures that interfere with light and cause particular wavelengths to be amplified or attenuated, and are capable of producing iridescence and metallic appearance [1,2].

The blue coloration in many birds is produced by 3D quasi-ordered spongy $\beta$-keratin and air nanostructures found in the medullary cells of feather barbs. Two main classes of 3D barb nanostructures are known: (i) characterized by a complex network of air channels and (ii) close packing spheroidal air cavities [3]. In order to elucidate the precise physical mechanism responsible for the production of avian colours, Prum et al. employed a spatial Fourier transform of a TEM image of the microstructure present in feather barbs of various avian species [4,5], and Saranathan et al. obtained the Fourier transform by X-ray scattering [3]. They found a clear ring structure around the origin in wave vector space, which indicated that the colour is produced by constructive interference between light waves scattered coherently by the nanostructure. They also used the 2D Fourier power spectrum (FPS) to simulate the reflected response. This method is based on the assumption of single scattering, i.e., light is scattered only once by the structure. The Fourier spectrum reveals the existence of a dominant length scale, which results in one peak in the predicted reflectance spectra [3-5].

However, the measured reflectance spectra of many bird species, including the Swallow Tanager (Tersina viridis), exhibit two peaks [3,6-8]. Even though the primary peak has been quite accurately reproduced, the FPS does not provide information about the secondary peak, whose origin has been attributed to multiple scattering effects [6].

To further investigate the mechanisms involved in the generation of birds' coloration, a rigorous electromagnetic method which takes into account the detailed geometry of the microstructure and the refractive indices of the constituent materials could be applied. In particular, the Korringa-Kohn-Rostoker (KKR) method appears as an interesting alternative for the calculation of the electromagnetic response of composite periodic structures made of spheres. Within this method, the electromagnetic interactions between the spherical scatterers are calculated by means of the layer-multiple scattering method [9-12]. The whole structure is considered as a stack of parallel layers formed by spheres periodically arranged in a 2D Bravais lattice. To solve the electromagnetic problem, the multiple scattering between spheres of each layer is calculated first, and then, the scattered response of multiple layers is determined by using a 
procedure similar to the one used to calculate the reflection and transmission properties of stratified media with planar interfaces.

In this paper we investigate the reflected response of the microstructure present in the feather barbs of the Swallow Tanager bird, by means of two different approaches. In Sec. 2 we describe the microstructure responsible for the blue coloration. This characterization is essential for the application of any simulation method. In particular, in Sec. 3 we briefly describe the FPS method and apply it to obtain a first approximation to the reflectance spectrum. In Sec. 4, the rigorous KKR method is used to compute the electromagnetic response of the structure, and results for different radii of the spheres and for different distances between adjacent layers are shown. Finally, concluding remarks are given in Sec. 5 .

\section{Characterization of the samples}

In Fig. 1 a) we show an image of a male Swallow Tanager which highlights the blue coloration of the feathers. The Swallow Tanager species exhibits high sexual dichromatism, males have blue plumage and females green. Also, males show an unusual optical property: from the human perspective, their colour changes as the angle between the directions of incidence and observation is varied. However, the hue practically remains unchanged if the orientation of the feather in relation to the observer or to the light source is modified $[7,8]$.

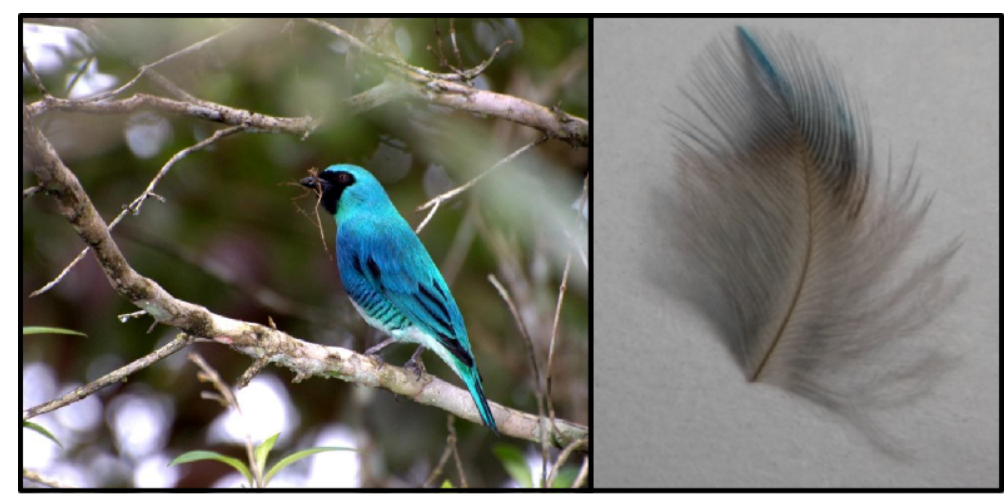

a)

b)

Fig. 1: a) Male Tersina viridis individual (https://commons.wikimedia.org/wiki/File:Tersina_viridis_male.jpg). b) Shoulder feather of the Tersina viridis sample under study. This specimen belongs to the Museo Argentino de Ciencias Naturales "Bernardino Rivadavia” (collection code MACN-Or-32133).

An image of a shoulder feather (collection code MACN-Or-32133) is shown in Fig. 1 b), where it can be observed that barbs closer to the most external part of the feather are blue whereas barbs in other regions exhibit a greyish coloration. Structural colour in birds' plumage is produced by the interaction of light with the photonic nanostructures present within the barbs [3]. Therefore, we are going to analyze the microstructure of the blue barbs of different feathers.

In order to characterize the samples of Tersina viridis, we made micrometric transversal cuts on different parts of the barbs following the protocol used by Barreira et al. [13]. Materials were mounted on copper grids to obtain images of the nanostructure using a transmission electron microscope (TEM).

Figs. 2 a) and b) show TEM images of transversal cuts at the extreme of a barb extracted from the most external part of the feather, which exhibit blue coloration (see Fig. 1 b)). The image in Fig. 2 b) shows details of the arrangement that produces colour in this species. A keratin cortex surrounds a threedimensional, quasi-ordered nanostructure composed of spherical air cavities immersed in $\beta$-keratin (refractive index, $\mathrm{n}_{\beta \text {-keratin }}=1.58 \pm 0.01$ [3]). Due to the fragility of the material, big white holes are observed in the spongy matrix, which are a result of the cutting procedure. A transversal cut of a barb from the base of the feather, which does not exhibit any colour effect, can be seen in Fig. 2 c). In this case the cortex can still be observed, although the spongy matrix with air cavities is absent. 


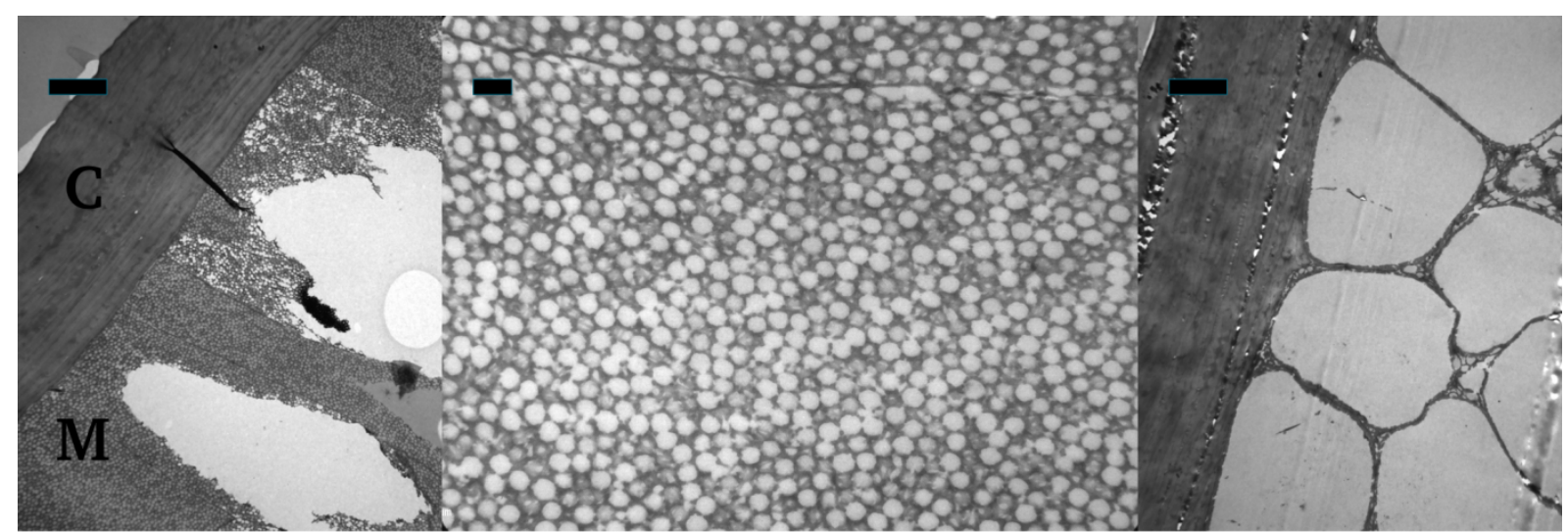

a)

b)

c)

Fig. 2: TEM images of transversal cuts of barbs of a male Tersina viridis specimen. a) Blue barb cut, composed of a spongy matrix (M) bounded by a keratin cortex (C). b) Zoomed view of the spongy region. c) Grey barb cut. Scale bars: (a), (c) $4 \mu \mathrm{m}$ and (b) $0.4 \mu \mathrm{m}$.

To obtain the geometrical parameters of the structure, we performed an exhaustive study of several TEM images using the ImageJ software [14] together with a statistical analysis. Each pixel in the TEM images can be assigned a dimensionless integer value in a gray scale from 0 (black) to 255 (white). Using the ImageJ software, the images were binarized, i.e., white or black colour was assigned to each pixel to identify and separate the air bubbles from the background. Then, we calculated the area and the position of the bubble regions in the analyzed images. In Fig. 3 a) we show an histogram of the air holes areas, from which the mean value of a single bubble area and its standard deviation results to be $23400 \pm 7300 \mathrm{~nm}^{2}$. Using this result and considering cavities with perfectly circular cross section, the value of the spheres' radius was estimated at $86 \pm 13 \mathrm{~nm}$. Also, the distance between nearest neighbours, defined as the average distance between the centre of a given sphere and the centre of all its nearest neighbours, was calculated. The results are shown in Fig. 3 b); the average nearest neighbour distance obtained is $a=240$ $\pm 19 \mathrm{~nm}$. A similar analysis was performed to estimate the number of nearest neighbours, which yielded a value of $5.5 \pm 1.7$, and to determine the angle between two consecutive nearest neighbours: $55 \pm 25^{\circ}$.

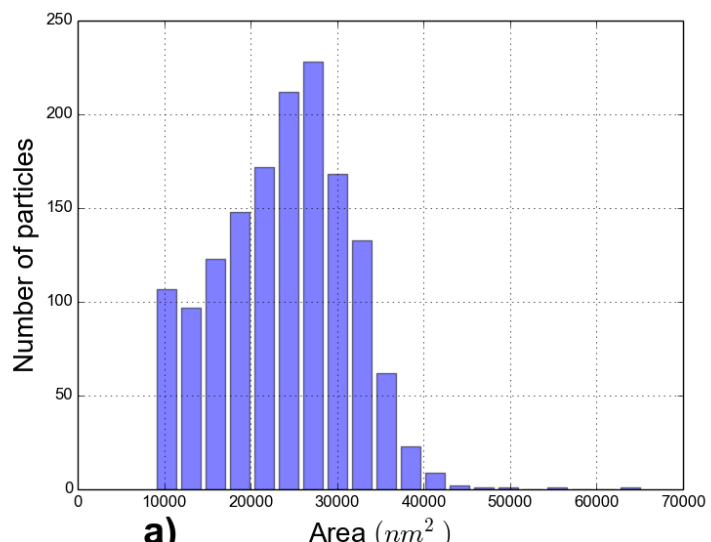

a)

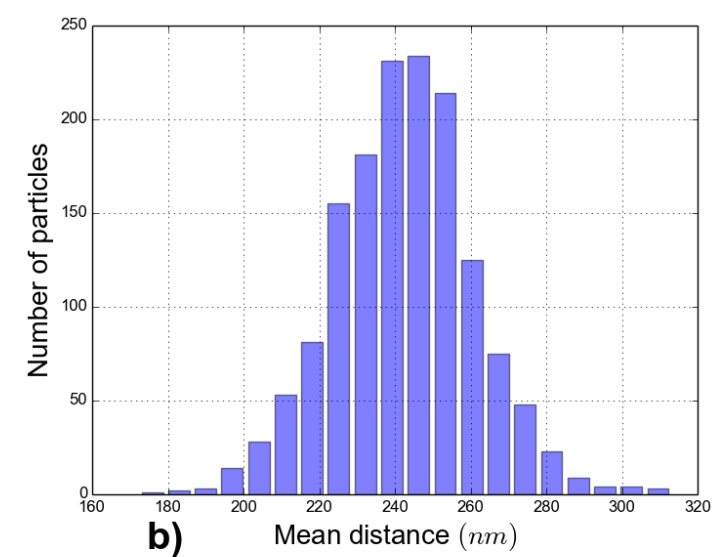

Fig. 3: Histograms: a) Area distribution of the air cavities in the binarized images. b) Average distance to the nearest neighbours.

\section{Fourier method}

The gray levels in the TEM images of the nanostructure represent variations in the refractive indices of the materials. However, it is a difficult task to extract from these images information about the periodicity of the structure. To do so, we analyzed them by applying the 2D Discrete Fourier Transform (DFT). Discrete Fourier analysis is a well known analytical tool that transforms a sample of data points into a 
mathematically equivalent sum of component sine waves of different frequencies and amplitudes. The amplitudes of each of the sine waves within the Fourier transform express the relative contributions of each frequency of variation to the periodicity of the original data. The plot of the variation in the squared amplitudes over all Fourier components is called the Fourier power spectrum (FPS). In this case, the frequencies are spatial frequencies and describe the spatial variations of the sample. In addition, the frequencies are equidistantly discretized, the step is inversely proportional to the total length of the sample and the number of frequencies is equal to the number of data points [15]. We apply the twodimensional case in which the discretized spatial frequencies are $q_{x}$ and $q_{y}$, and are written as integer multiples of the step: $q_{0 x}=2 \pi / L_{x}$ and $q_{0 y}=2 \pi / L_{y}$; where $L_{x}$ and $L_{y}$ are the actual width and height of the TEM image, respectively. Then, after applying the DFT, we obtained the FPS of the TEM images.

We calculated the FPS for the TEM images from different parts of the barb with 20000x, 30000x and $50000 x$ magnifications, which correspond to $L_{x}=7074 \mathrm{~nm}, 4656 \mathrm{~nm}$ and $2820 \mathrm{~nm}$ and $L_{y}=5233 \mathrm{~nm}$, $3445 \mathrm{~nm}$ and $2086 \mathrm{~nm}$, respectively. As an example, in Fig. 4 we show the FPS obtained from Fig. 2 b). The spatial frequencies investigated range between $0 \mathrm{~nm}^{-1}$ and $0.08 \mathrm{~nm}^{-1}$, which correspond to the visible and to the near UV parts of the spectrum. In order to highlight the relevant features of the image, we plot $\log (1+\mathrm{FPS})$.

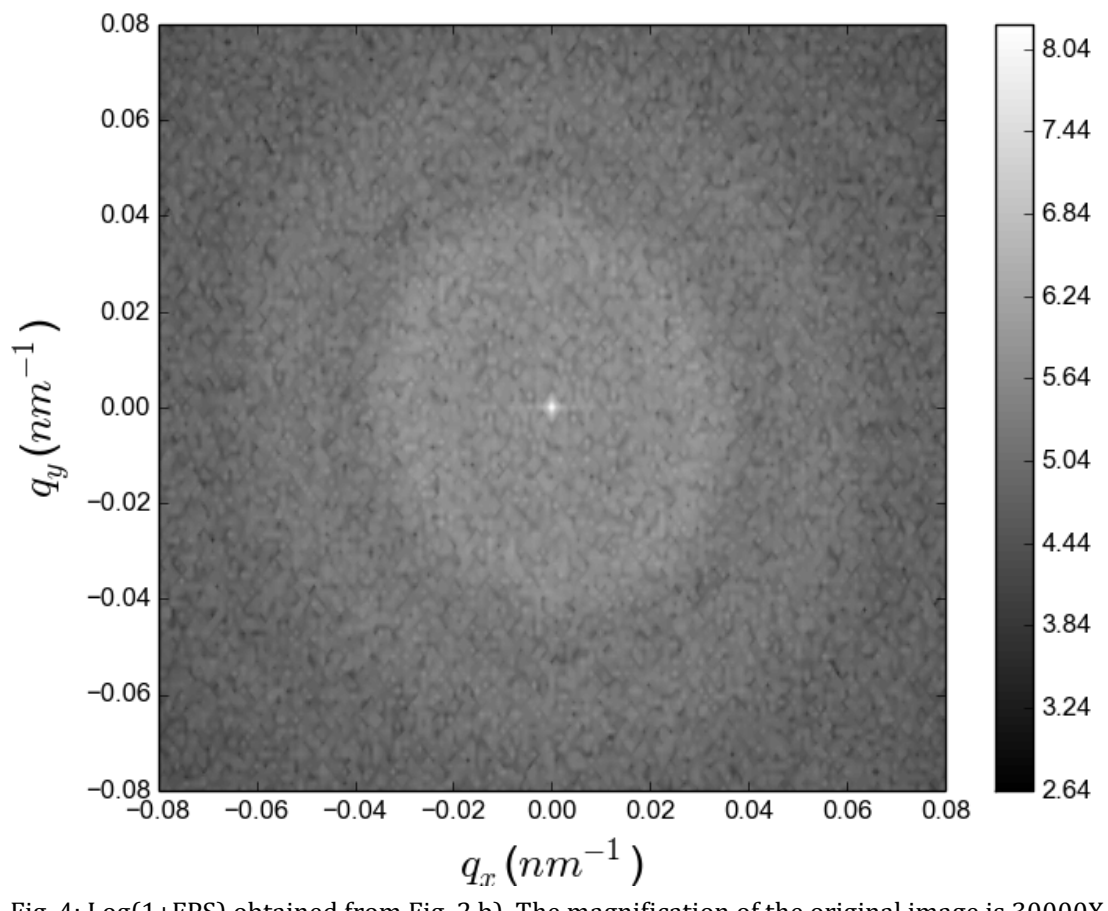

Fig. 4: $\log (1+\mathrm{FPS})$ obtained from Fig. 2 b). The magnification of the original image is $30000 \mathrm{X}$

As observed, the calculated spectra exhibit a ring-shape. This distribution of spatial frequencies reveals that the nanostructure is a quasi-ordered array of spheres, and therefore, it can produce coherent scattering. Averaging the intensities of every concentric circle, we obtain the radial mean intensity $(I(q))$ for each spatial frequency $q\left(q^{2}=q_{x}{ }^{2}+q_{y}{ }^{2}\right)$. In Fig. 5 we show curves of $I(q) / I\left(q_{\max }\right)$, where $q_{\max }$ is the frequency of the highest peak, for several TEM images with different magnifications. 


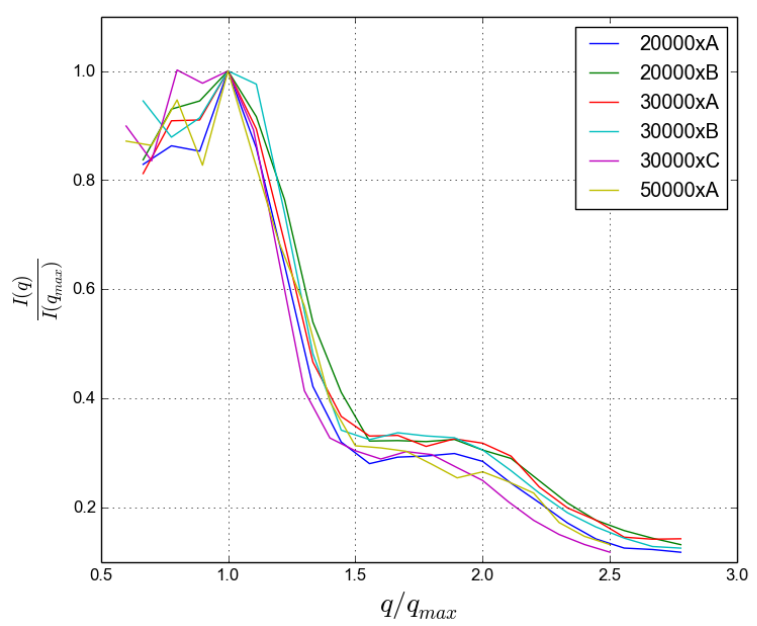

Fig. 5: Normalized radial mean intensity $I(q) / I\left(q_{\max }\right)$ vs. normalized spatial frequency $q / q_{\max }$ for different TEM images and magnifications.

In Fig. 6 a) we show the average value of the curves shown in Fig. 5. This curve was deconvolved into two Gaussian functions to find the position of the peaks, their relative intensity and the full width at halfmaximum (FWHM). The nearest-neighbour spacing can be estimated from the position of the highest peak and the FWHM, reaching a value of $222 \pm 73 \mathrm{~nm}$. It is noteworthy that this result is in agreement with the estimation obtained in Sec. 2 by direct analysis of the TEM images.
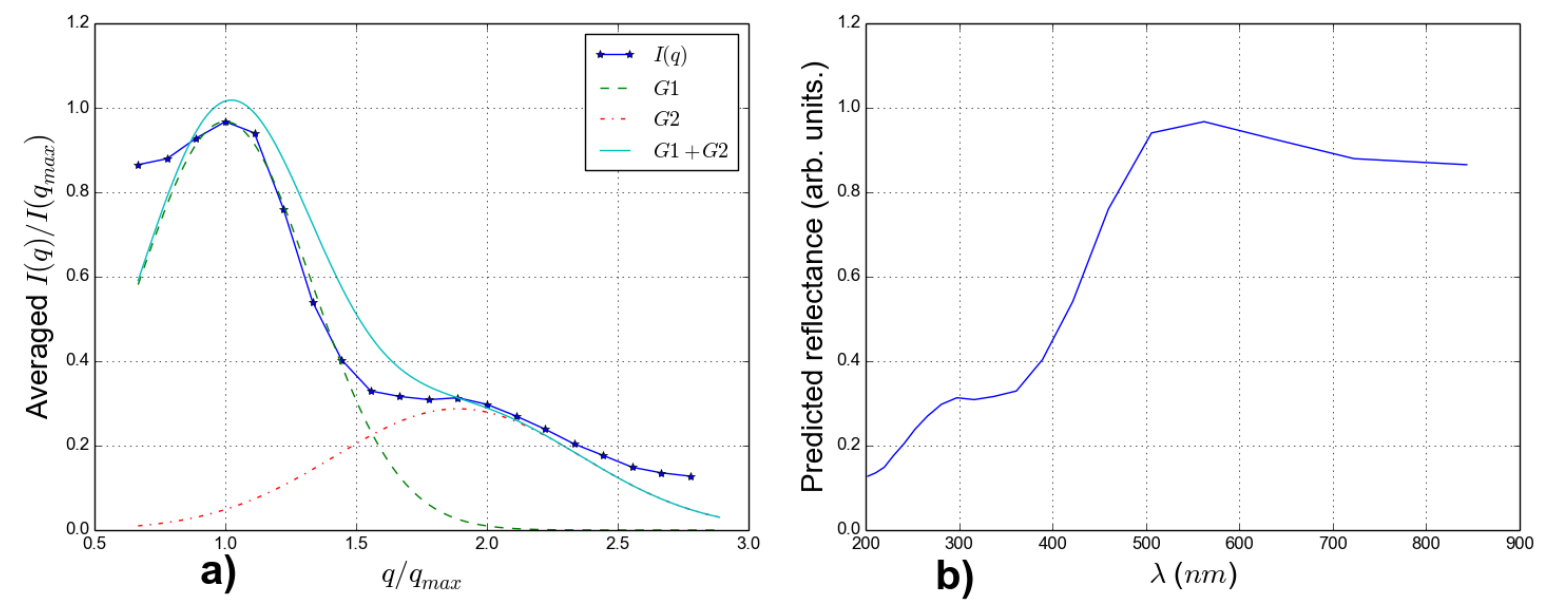

Fig. 6: a) Averaged $I(q) / I\left(q_{\max }\right)$. The spectrum was approximated by the superposition of two gaussian functions, G1 and G2. b) Reflectance spectrum predicted using Eq. (1).

As described in $[3,15]$, the radial mean intensity can be used to predict the optical reflectance spectra using single-scattering theory by mapping the intensities from spatial frequency $(q)$ to wavelength $(\lambda)$ space. This relationship follows from Bragg's law, which for normal incidence of light and back-scattering geometry reads:

$$
\lambda=2 \mathrm{n}_{\mathrm{avg}}(2 \pi / q) .
$$


In the expression above, $\mathrm{n}_{\text {avg }}$ is the average ${ }^{\circ}$ or effective refractive index, which for this sphere-type nanostructure has been estimated at $\approx 1.265$ [6].

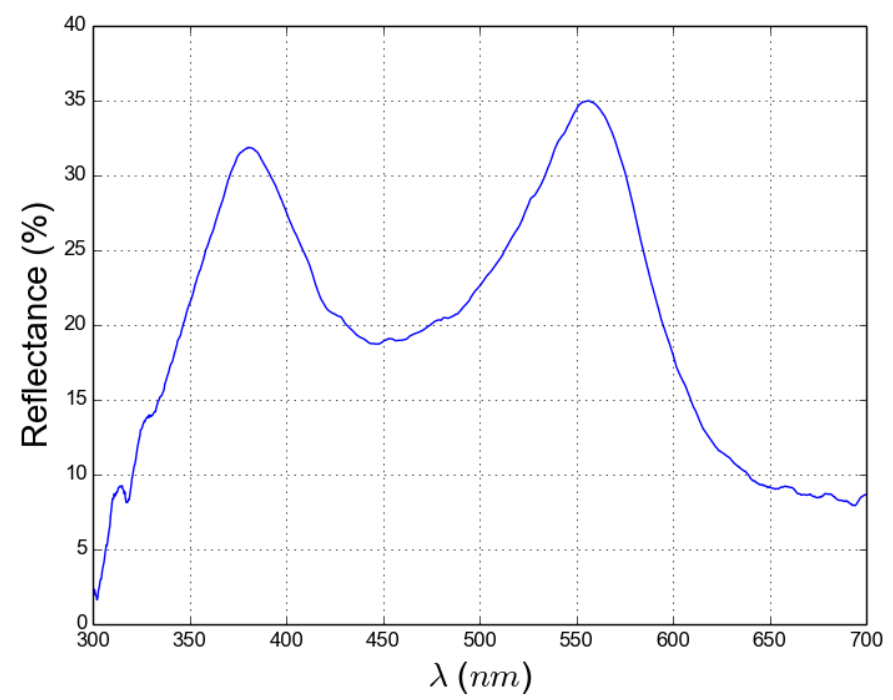

Fig. 7: Normal incidence reflectance spectrum obtained by averaging 15 reflectance measurements performed on different parts of the shoulder of the sample under study.

In Fig. 6 b) we plot the predicted reflectance spectrum, which is obtained from Fig. 6 a) by transforming variable q into $\lambda$ using equation (1). As observed, the highest reflectance peak appears at $562 \mathrm{~nm}$. This result is in very good agreement with the measured incidence reflectance curve (Fig. 7), which shows a characteristic bimodal reflectance profile with a primary reflectance peak at $557 \mathrm{~nm}$ and an additional peak in the ultraviolet region with a lower intensity. The curve in Fig. 7 has been obtained by averaging 15 normal incidence reflectance spectra corresponding to different sections of the shoulder of the same investigated specimen. The method employed to perform the reflectance measurements is described in [13].

However, as mentioned above, the FPS is an approximate method which cannot reproduce higher order features in the reflected response, such as multiple scattering effects. Therefore, in Sec. 4 we apply a rigorous electromagnetic method which takes into account the detailed geometry of the microstructure and the refractive indices of the constituent materials to further investigate the mechanisms involved in the generation of birds' coloration.

\section{KKR method}

As mentioned in Sec. 1, the KKR method allows us to calculate the reflectance and transmittance of a 3D photonic crystal formed by a periodic arrangement of spheres immersed in a host medium [9-12,16]. If the distribution of air spheres within the spongy matrix of Tersina viridis barbs is considered as a regular structure, one can use the numerical implementation of the KKR method developed by Stefanou et al. [12] (MULTEM2) to compute the electromagnetic response of the analyzed structures. For this purpose, not only the geometrical distribution of spheres, but also the constitutive parameters of the host material should be known. According to the characterization of the samples described in Sec. 2, the number of nearest neighbours is $5.5 \pm 1.7$ and the angle between two consecutive nearest neighbours is $55^{\circ} \pm 25^{\circ}$. These values suggest that the arrangement of spheres resembles a hexagonal close-packed lattice (hp) [17]. The primitive cell of such arrangement is shown in Fig. 8 a). We modelled the structure as a multilayer of 34 parallel identical slabs of air spheres of radius $r$ immersed in a $\beta$-keratin matrix. The centres of all the spheres within a given slab are contained in a plane parallel to the slabs, and the distribution of spheres within each layer corresponds to a hexagonal lattice, as schematized in Fig. 8. The distance $z$ between two consecutive layers is kept equal for all the structure; however, each layer is laterally displaced with respect to its neighbour layers, as shown in Fig. 8 b) and c), where the red and the cyan circles represent two adjacent layers of spheres. 


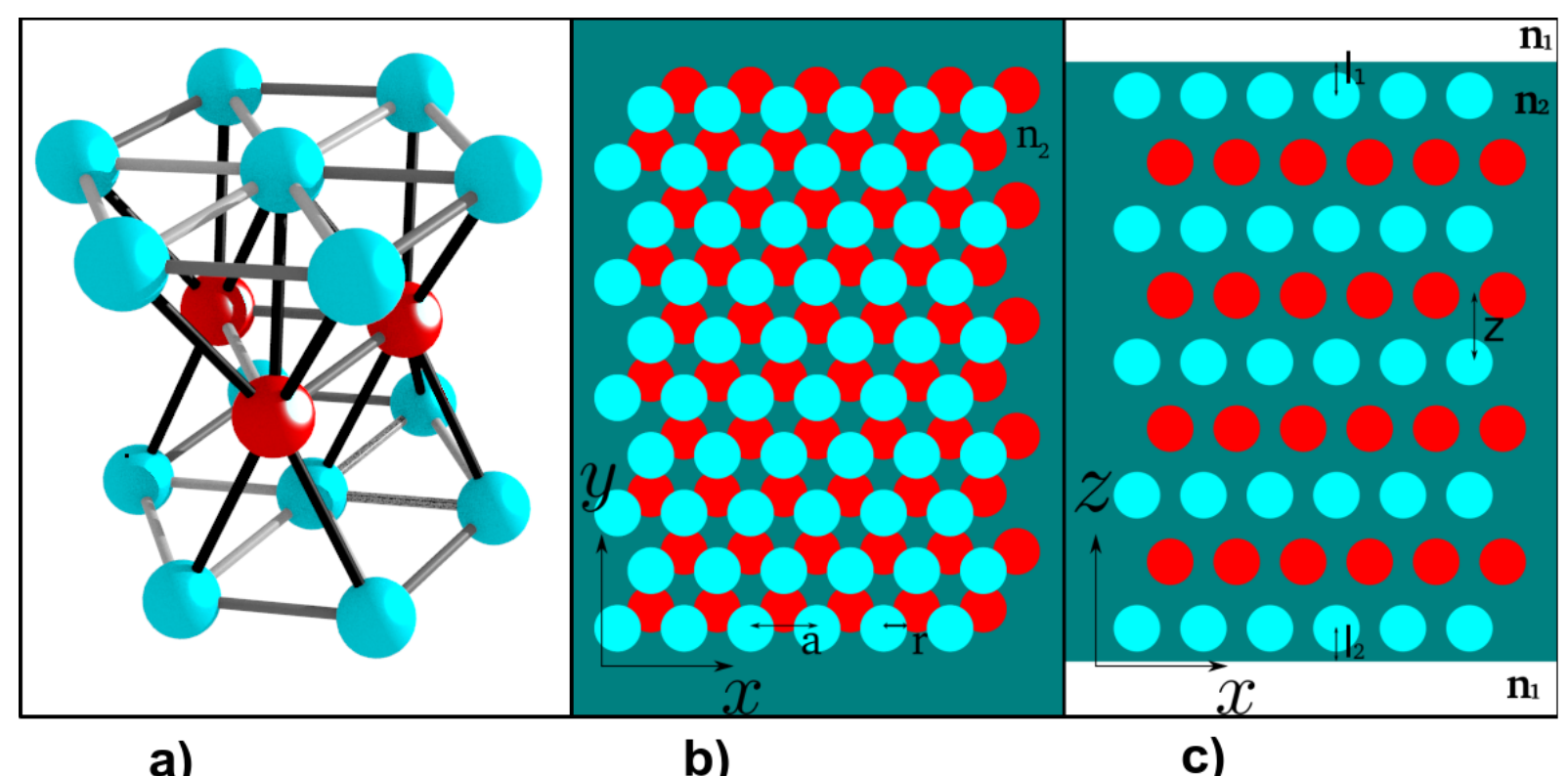

Fig. 8: Hexagonal close-packed lattice considered for the KKR calculations. a) 3D view; b) Top view; c) Front view.

Even though the structure is infinitely periodic in the $x$ and $y$ directions, it is finite in the $z$ direction. The boundary surfaces with the external medium (air) are planes parallel to the slabs, above and below the structure. The distance $l_{1}\left(l_{2}\right)$ represents the vertical distance between the upper (lower) contact surface and the centre of a sphere in the first (last) slab (see Fig. $8 \mathrm{c}$ )). Then, the geometrical parameters of the structure required to feed the numerical code are $r, a, z, l_{1}$ and $l_{2}$. Besides, the constitutive parameters of the materials involved should be provided.

We considered a normally incident TE-polarized plane wave of wavelength $\lambda$ and employed the MULTEM2 program to compute the reflectance spectra for different values of the geometrical parameters. The range of wavelengths considered covers the visible and the near-UV spectrum, since we aim at investigating the structural colour effects as well as the UV response of the samples. The values $l_{1}$ and $l_{2}$ were fixed at $a / 2$, which ensures that for all the chosen configurations the spheres remain completely immersed in the $\beta$ keratin medium. The air spheres have $\varepsilon=1$ and $\mu=1$, and for the $\beta$-keratin we considered $\varepsilon=2.4336$ and $\mu=1$. The lattice parameter $(a)$ and the radius of the spheres $(r)$ were estimated from the analysis of TEM images, and in the numerical examples we considered $a=240 \mathrm{~nm}$ and $r=86 \mathrm{~nm}$, which correspond to the respective mean values obtained in Sec. 2 .
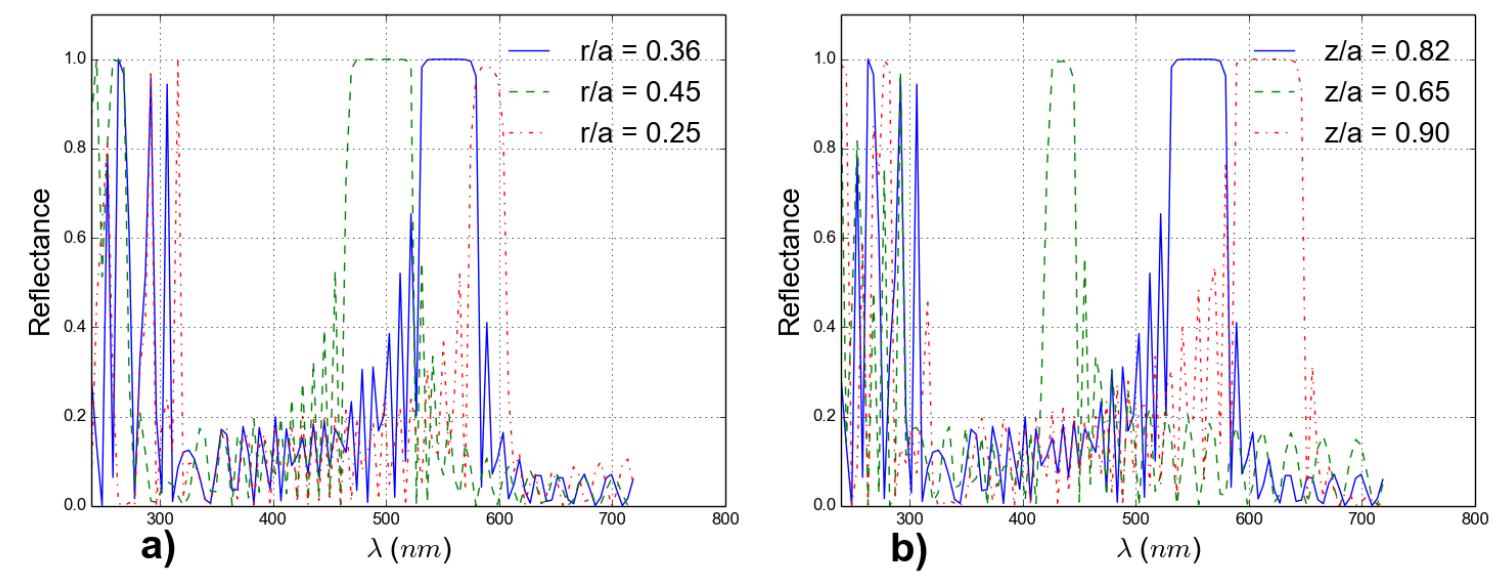

Fig. 9: Optical reflectance spectra for a multilayer hexagonal close-packed array (lattice constant $a=240 \mathrm{~nm}$ ) of air spheres in a $\beta$ keratin matrix ( $\varepsilon=2.4336$ and $\mu=1$ ), considering 34 layers of spheres. a) $z=\sqrt{2 / 3} a$ and $r$ is varied; b) $r=86 \mathrm{~nm}$ and $z$ is varied. 
In Fig. 9 we show the reflectance spectra obtained for different values of the sphere's radius $r$ and of the distance between adjacent layers $z$. In Fig. 9 a) we fixed $z=\sqrt{2 / 3} a$, and $r$ was varied around its mean value, whereas in Fig. 9 b) $r=86 \mathrm{~nm}$ and $z$ was varied.

The position of the peak within the visible range for the reference configuration in Fig. $9(r=86 \mathrm{~nm}$ and $z=\sqrt{2 / 3} a$ ), approximately at $\lambda=560 \mathrm{~nm}$, is in very good agreement with that obtained with the FPS method (see Fig. 6b) and also with the measurement (Fig. 7), although it can be noticed that the position and the width of the peak depend on the parameters $z$ and $r$.

It is important to note that the theoretical method reproduces not only the intensification of the reflectance in the visible region of the spectrum but also the intensification in the UV range, which cannot be reproduced by the Fourier method. This is because the KKR is a full vector rigorous method that takes into account single scattering events as well as the effects of multiple scattering. However, it should be noted that the curves of Fig. 9 and the measured reflectance (Fig. 7) show differences, mainly in the shape of the peaks and in the reflectance values. These differences are to expect since the curves of Fig. 9 correspond to a perfectly periodic structure, and the biological structure is not strictly periodic.

The reflectance increment obtained in the UV range could be associated with the secondary peak observed in the measured reflectance spectrum, which has been attributed to multiple scattering effects [6].

\section{Conclusions}

We investigated the structural colour generation in male Swallow Tanager individuals. Barbs from the most external part of the feather have been analyzed and characterized by transmission electron microscopy, which revealed a microstructure consisting of quasi-spherical air cavities embedded in a $\beta$ keratin matrix. To compute the electromagnetic response of the structure, two different theoretical approaches have been applied. First, we used a simplified model based on Fourier analysis, to calculate the spectral position of the dominant reflectance peak in the visible range of the spectrum. Then, we applied the KKR method to obtain the reflectance of the structure taking into account their relevant geometrical parameters and the refractive indices of the constituent materials. We performed numerical simulations varying the spheres' radius and the typical distances of the 3D lattice. The results confirm that the interaction of light with the microstructure of the barb produces coherent scattering and this is the underlying mechanism responsible for the observed colour. Besides, the KKR curves exhibit an enhancement in the UV region, which is in agreement with the measured reflectance of Swallow Tanager. These results evidence the potential of the KKR formalism to accurately reproduce the electromagnetic response of natural structures and to reveal features that cannot be explained using the Fourier approach.

However, since the actual biological structure has certain degree of disorder (the air bubbles are not perfectly spherical, the arrangement is not strictly a hexagonal lattice, etc.), in order to reproduce more precisely its electromagnetic response it is necessary to take into account these deviations within the theoretical model. As noticed by other authors [18,19], within the UV region, the KKR method is more sensitive to the disorder present in the analyzed structure, and then, a more careful treatment should be carried out to accurately account for the details of the actual biological structure under study.

\section{Acknowledgements}

D. S. and M. I. acknowledge partial support from Consejo Nacional de Investigaciones Científicas y Técnicas (CONICET PIP 112-201101-00451) and Universidad de Buenos Aires (UBACyT 20020150100028BA). C. D. acknowledges an UBA scholarship. 